\title{
L'enseignement des sciences au secondaire obligatoire en Suisse romande, au regard des enquêtes internationales sur la culture scientifique des jeunes
}

\section{Laura Weiss}

PISA 2006 a évalué la culture scientifique des jeunes de 57 pays. A une époque où tous les pays de l'OCDE voient une désaffection des jeunes vis-à-vis des sciences et des compétences des élèves généralement peu satisfaisantes dans ce domaine, il est crucial d'estimer la contribution de l'école, et singulièrement de l'école obligatoire, à cette culture. Dans cet article, nous inventorions les curriculums des sciences au secondaire obligatoire par rapport aux contenus des questions PISA pour comparer l'enseignement scientifique prescrit aux injonctions des experts se préoccupant de favoriser l'intérêt des jeunes vis-à-vis des sciences. Nous brossons ainsi un tableau de l'enseignement des sciences des sept cantons en Suisse romande.

\section{Introduction}

En août 2010, le Conseil fédéral rend un rapport en réponse à une série de cinq postulats ${ }^{1}$ de parlementaires fédéraux à propos de l'«ampleur et [des] causes de la pénurie de personnel qualifié dans les domaines MINT (mathématiques, informatique, sciences naturelles et technique)»(Conseil fédéral, 2010). Il y est constaté que «les intérêts, et de manière associée l'orientation professionnelle des jeunes, sont fixés dans une large mesure déjà à la fin de la scolarité obligatoire. Dans ce contexte, un rôle clé revient aux mathématiques, à la physique et à l'intérêt pour la technique en général. Les jeunes de quinze ans qui sont intéressés par les disciplines MINT y obtiennent de bonnes notes et jugent leurs compétences bonnes ont une probabilité accrue d'entreprendre plus tard des études MINT.» (Conseil fédéral, 2010, p. 3). Dans ses recommandations, «le Conseil fédéral juge indispensable une promotion permanente de la compréhension technique. C'est pourquoi il faut stimuler l'intérêt pour les domaines MINT à l'âge préscolaire, au jardin d'enfants et aux degrés primaire et secondaire I, et poursuivre de façon rigoureuse les actions déjà entreprises dans ce sens par les Académies et les hautes écoles» (Conseil fédéral, 2010, p. 4). Ces préoccupations ne sont pas nouvelles. Déjà en 2000, une parlementaire déposait le postulat 
«Renforcer l'intérêt pour l'étude des branches scientifiques ${ }^{2}$ » qui, conjointement à un autre postulat sur le problème de la question genre en mathématiques ${ }^{3}$, avait donné lieu à un mandat de la Conférence suisse de coordination pour la recherche en éducation (CORECHED) au Centre suisse de coordination pour la recherche en éducation (CSRE). Le résultat de cette étude (Coradi, Denzler, Grossenbacher \& Vanhooydonck, 2003) et les ouvrages qu'elle recense posent plus largement la problématique de la désaffection envers les sciences dans tous les pays développés. Ces conclusions rejoignent celles de plusieurs rapports internationaux, en particulier le Rapport Rocard (Rocard et al., 2007) réalisé pour la Commission européenne qui attribue à l'enseignement des sciences à l'école une part importante de la responsabilité de la baisse de l'attractivité des sciences envers les jeunes.

La publication en 2007 (CDIP, 2007, 2009; Nidegger, 2008; OCDE, 2007) des résultats de l'enquête PISA 2006 centrée sur la culture scientifique apporte d'autres informations sur ce sujet. En effet, cette enquête tente d'une part d'évaluer l'attitude des jeunes de 15 ans envers les sciences et d'autre part de mesurer leurs connaissances et compétences scientifiques. Sur ce deuxième point, la Suisse obtient au test PISA sciences une moyenne honorable ( 512 points à comparer avec la moyenne OCDE fixée à 500 points), mais les élèves de Suisse romande sont moins performants que leurs camarades alémaniques (502 points) et certains cantons romands obtiennent une moyenne inférieure aux fatidiques 500 points. Ces résultats, peu satisfaisants au regard des ambitions en sciences d'un pays qui compte deux écoles polytechniques fédérales mondialement reconnues, pourraient être interprétés comme un enseignement des sciences déficient à l'école obligatoire puisque PISA teste les élèves à la fin de celle-ci.

Pour apporter un éclairage plus objectif à ce débat, nous procédons en deux étapes. Nous analysons d'abord l'organisation de l'enseignement scientifique dans les sept cantons suisses romands et les prescriptions officielles de cet enseignement définies par les curriculums, pour déterminer dans quelle mesure y sont prises en compte les recommandations à l'intention des institutions scolaires des différents rapports qui se penchent sur la désaffection des jeunes envers les sciences. Nous nous intéressons plus précisément aux contenus de l'enseignement de physique-chimie, ces deux branches étant jumelées dans les cantons romands à l'école obligatoire. Nous étudions ensuite les questions de PISA 2006 visant à évaluer la culture scientifique, pour établir dans quelle mesure il y a corrélation entre celles-ci et les curriculums romands, en particulier en physique-chimie. Mais pourquoi l'OCDE à travers ses enquêtes PISA veut-elle tester la culture scientifique et qu'entend-elle par cette expression? 


\section{Cadrage théorique}

\section{La culture scientifique selon PISA}

L'OCDE a lancé depuis 2000 les enquêtes cycliques PISA pour évaluer, de façon comparative entre les pays, la culture et les attitudes des jeunes de 15 ans envers différents domaines de la connaissance humaine. Son objectif n'est pas tant de tester les connaissances scolaires au sortir de l'école obligatoire, mais plutôt d'apporter un certain nombre de réponses à des questions générales comme: les jeunes sont-ils bien préparés à relever les défis que l'avenir leur réserve? Sont-ils prêts à être des membres productifs de l'économie et de la société? Sont-ils capables d'analyser, de raisonner et de transmettre correctement leurs idées? Détiennentils les connaissances et compétences essentielles pour pouvoir participer pleinement à la vie de la société? En ce qui concerne les sciences, le rapport «PISA 2006. Les compétences en sciences, un atout pour réussir» (OCDE, 2007) part aussi du même constat inquiétant des parlementaires nationaux cités ci-dessus, à savoir que le pourcentage d'élèves qui s'orientent vers des études scientifiques ou technologiques a diminué dans tous les pays de l'OCDE. En outre, il considère que l'importance de la technologie dans notre société impose que les futurs adultes comprennent des théories et des concepts scientifiques fondamentaux et sachent appréhender et résoudre des problèmes scientifiques. C'est pourquoi le troisième cycle PISA en 2006 a porté principalement sur les compétences des élèves en sciences et leur intérêt pour les domaines scientifiques, évaluant ainsi leur culture scientifique («scientific literacy»). Par culture scientifique, PISA entend:

La mesure dans laquelle un individu [de 15 ans]:

- possède des connaissances scientifiques et les applique pour identifier des questions, acquérir de nouvelles compétences, expliquer des phénomènes de manière scientifique et tirer des conclusions fondées sur des faits à propos d'aspects scientifiques, par exemple en distinguant les faits scientifiques de ceux qui ne le sont pas;

- comprend les éléments caractéristiques des sciences en tant que forme de recherche et de connaissance humaines;

- est conscient du rôle des sciences et de la technologie dans la constitution de notre environnement matériel, intellectuel et culturel;

- a la volonté de s'engager en qualité de citoyen réfléchi dans des problèmes à caractère scientifique et touchant à des notions relatives aux sciences.

La culture scientifique passe par la compréhension de concepts scientifiques et renvoie à la capacité d'appliquer une perspective scientifique et d'analyser les faits de manière scientifique. (OCDE, 2007, p.23) 
À partir de ces prémisses, les contenus des questions de l'enquête PISA 2006 ont été construits avec deux objectifs. Premièrement, les questions visent à évaluer les connaissances des élèves «en» sciences, c'est-à-dire à propos des théories et des concepts scientifiques fondamentaux dans quatre grands domaines scientifiques appelés systèmes: systèmes physiques, systèmes vivants, systèmes de la Terre et de l'univers et systèmes technologiques, qui correspondent aux champs classiques de l'étude des sciences de la nature. Deuxièmement, PISA s'attache à tester des connaissances «à propos» des sciences, déclinées d'une part en démarche scientifique et d'autre part en explication scientifique, précisées dans le tableau 1.

\section{Tableau 1: Les catégories de connaissances «à propos» des sciences de PISA 2006} (OCDE, 2007, p. 44)

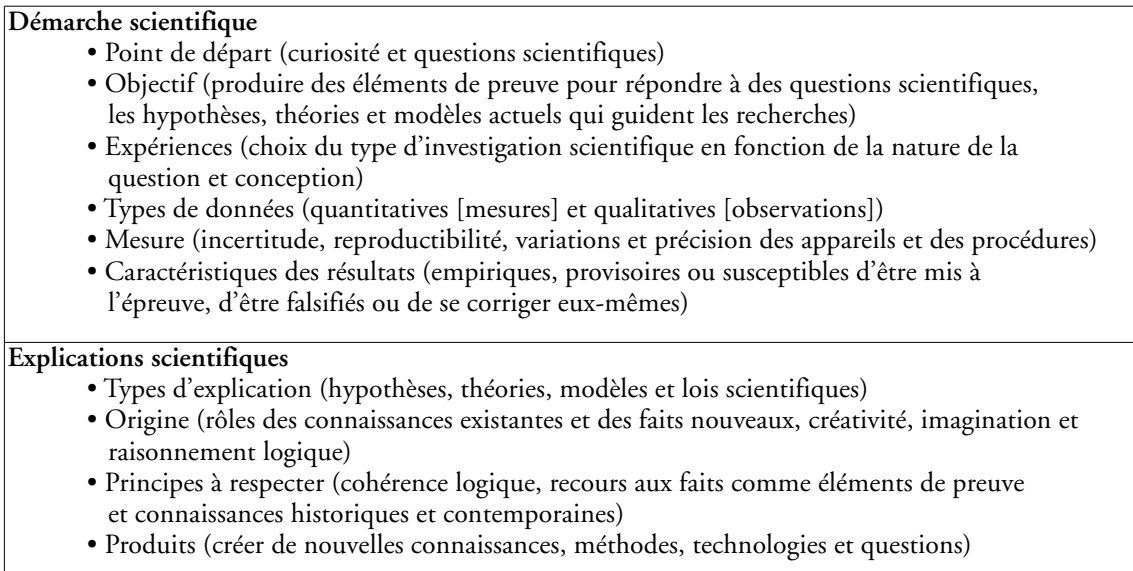

Cette distinction entre connaissances «en» et «à propos» des sciences est fondamentale pour notre propos, parce qu'elle rejoint l'un des reproches qui sont faits à l'enseignement scientifique: la trop grande insistance sur les connaissances livresques au détriment de la formation à une posture scientifique.

\section{Le problème de la motivation vis-à-vis des sciences}

Il existe de très nombreuses publications à propos de la désaffection des jeunes vis-à-vis des sciences et prônant des mesures pour l'enrayer. Pour nous concentrer sur la Suisse, nous nous appuyons d'abord sur le rapport de tendance CSRE n6 (Coradi et al., 2003), rédigé suite au mandat de la CORECHED. Celui-ci synthétise les résultats suisses de recherches et d'enquêtes internationales dont PISA $^{4}$ et TIMSS 5 et identifie parmi les facteurs externes 6 influençant les compétences en sciences, d'une part les attentes des parents et l'influence des groupes de pairs et d'autre part les caractéristiques de l'enseignement, dont les stéréotypes des enseignants et les formes privilégiées par l'école pour l'enseignement et 
l'apprentissage. Cette étude rend responsable de la baisse de l'attractivité des études scientifiques, entre autres, l'«inadéquation du cadre scolaire et de l'approche didactique et méthodologique de l'enseignement, qui ne sont aptes ni à éveiller ni à renforcer l'intérêt des jeunes pour les disciplines mathématiques, scientifiques et techniques.» (Coradi et al., 2003, p.11). Parmi les six recommandations auxquelles il aboutit, les troisième et quatrième recommandations, à savoir «Promouvoir le développement de l'enseignement» et "Améliorer le programme et l'organisation de l'enseignement», associent les performances en sciences plutôt moyennes en comparaison internationale des élèves suisses avec le constat que l'enseignement scientifique commence relativement tard en Suisse et que celui des branches techniques demeure marginal. Le rapport préconise de:

Renforcer la formation de base dans les domaines scientifiques et techniques [qui] devrait intervenir dès le début de la scolarité obligatoire [...]. Il convient aussi de trouver une solution (module intégré aux disciplines existantes ou projet interdisciplinaire, par exemple) pour améliorer la compréhension de la technique [afin de] stimuler la curiosité et l'intérêt des élèves pour ces disciplines dès les premières années de la scolarité. (Coradi et al., 2003, pp. 14-15)

Pour étayer ces recommandations, les auteurs du rapport ont recensé une série d'études, en particulier allemandes, qui montrent que l'organisation de l'enseignement scientifique influe sur l'attractivité des sciences pour les élèves. Sont relevés le manque d'un enseignement de la technique (Pfenning, Renn \& Mack, 2002) et la forme scolaire trop rigide divisant l'enseignement scientifique en disciplines distinctes et en cours de 45 minutes (Lechner, 1994) et préconisées une réorganisation structurelle permettant un enseignement par trimestre ou semestre au rythme de deux heures de suite par semaine et la mise en œuvre de méthodes de travail scientifiques, ce qui favorise le transfert de connaissances et de compétences d'une discipline à l'autre (Coradi et al., 2003). La mise en cause de l'organisation de l'enseignement des sciences n'est cependant pas circonscrite à l'Allemagne. Le Haut Conseil de l'Éducation (HCE) français recommande que le «socle commun des connaissances et des compétences» français comporte des compétences «en culture scientifique et technologique», favorisées «dans le cadre de la scolarité obligatoire, [par le fait de] décloisonner dans une large mesure les enseignements scientifiques et techniques» (Raulin, 2008, p. 25), et "en évitant la simple juxtaposition d'éléments des programmes disciplinaires, pour assurer la cohérence de l'enseignement scientifique.» (p. 26).

D'autres auteurs signalent les difficultés de mise en place d'une didactique adaptée aux disciplines scientifiques: Zwick et Renn (2000) reprochent à l'enseignement de la physique la place trop importante faite aux mathématiques qui le rend abstrait et théorique, ne prenant que marginalement en compte le champ de la technique et sans rapport avec la vie de tous les jours. Du coup, les élèves considèrent les branches scientifiques, particulièrement la physique et la chimie comme ennuyeuses, monotones et stériles. Durner (2002) s'inquiète de la quali- 
fication insuffisante des enseignants de sciences qui les rend incapables d'éveiller l'intérêt de leurs élèves en exploitant ce qu'il y a de passionnant dans la recherche scientifique et les découvertes fondamentales. Il regrette le manque de transposition systématique dans l'enseignement de la recherche en physique, en chimie, en biologie, ainsi que dans les domaines interdisciplinaires apparentés. Pour leur part, Giordan et De Vecchi (1994) soulignent la nécessité de prendre en compte les conceptions initiales des élèves pour les déconstruire, si on veut que l'apprentissage des sciences puisse être efficace. Mais un enseignement davantage basé sur un modèle constructiviste de l'apprentissage se heurte, en Suisse, selon Labudde (2000), aux habitudes d'enseignement de la physique laissant peu de place aux expériences-élève et aux débats scientifiques en classe, ainsi qu'au contenu trop vaste prévu par les plans d'études dans un temps d'enseignement restreint.

Pour lutter contre cet état de fait, le rapport Rocard (Rocard et al., 2007) donne comme exemples de réussite des expériences comme "La main à la pâte», «Pollen» ou «Sinus Transfer», qui, menées aussi bien au primaire qu'au secondaire, montrent le succès dans l'enseignement scientifique du passage d'une démarche déductive à une méthode basée sur le questionnement, l'Inquiry based science education (IBSE) ou Inquiry based learning (IBL), traduits souvent par démarche d'investigation, qui éveille l'intérêt des élèves et par suite leur taux de réussite en sciences. Si on reprend les caractéristiques des connaissances «à propos» des sciences de PISA 2006 reproduites dans le tableau 1, on peut constater la similitude entre la définition de la démarche scientifique selon PISA et de la démarche d'investigation.

\section{Le concept de curriculum}

Si l'idée d'organiser et de planifier les contenus de l'enseignement est bien plus ancienne, puisqu'on trouve par exemple à Genève des programmes d'enseignement à partir de la deuxième moitié du 19e siècle (Farquet, 1993), Crahay, Audigier et Dolz (2006) font remonter la recherche curriculaire à un article de Schwab de 1978 «What do scientists do?», mais soulignent que ce n'est que récemment que «le curriculum constitue un objet d'investigation scientifique et définit un champ d'études dont les contours restent flous malgré une littérature scientifique foisonnante.» (Crahay et al., 2006, p. 7). Distinguant trois axes thématiques principaux, premièrement la construction du curriculum comme objet de tensions entre une position dite technologique et une approche qui souligne les enjeux sociaux des changements curriculaires, deuxièmement la tension entre le monde des décideurs politiques et le monde de la pratique et troisièmement la question de la gestion des systèmes éducatifs par le pilotage par les outputs versus le développement de curriculums, ces auteurs ajoutent cependant une autre interrogation, à savoir "comment l'apprentissage des élèves est-il influencé par le curriculum?» (p. 8), qu'ils ont choisi de ne pas retenir «pour éviter l'important mais envahissant débat sur l'efficacité de l'école» (p. 8), tout en reconnaissant que cette question reste en arrière-plan omniprésente dans les analyses. 
En s'appuyant sur l'étymologie latine du terme curriculum, qui signifie courir dans le cadre d'une compétition, on est plutôt renvoyé à la suite des étapes dans le processus d'enseignement qui permettent aux élèves d'atteindre le but visé, l'apprentissage des contenus et des méthodes d'une discipline scolaire. Pour sa part, Perrenoud (1993) rappelle que «dans les pays anglo-saxons, on parle de curriculum pour désigner le parcours éducatif proposé aux apprenants, alors qu'en français on dira plus volontiers plan d'études, programme ou cursus, selon qu'on met plutôt l'accent sur la progression en les connaissances, les contenus successifs ou la structuration de la carrière scolaire» (p. 61). Parmi les nombreuses définitions de ce terme polysémique, nous retenons celle de Landsheere (1991) qui l'élargit à toutes les composantes de l'enseignement, des finalités jusqu'à l'évaluation, le distinguant ainsi du plan d'études ou du programme:

Tel qu'il entre dans l'usage français, l'emploi du terme curriculum marque la volonté de lier fonctionnellement les composantes de l'enseignement: intentions, contenus, organisation, méthodes, environnements humain et matériel (y compris les manuels scolaires), évaluation et dispositions relatives à la formation des enseignants. (p. 169)

\section{Problématique et méthodologie}

Selon les experts de l'enseignement scientifique, celui-ci doit être repensé pour développer l'intérêt des élèves pour les matières scientifiques, dont découleront à terme de bons résultats scolaires, ce qui favorisera une orientation ultérieure vers des études dans ce domaine. Du point de vue organisationnel, cette instruction doit commencer le plus tôt possible, avec les élèves les plus jeunes, et ne pas être distillée au rythme de 45 ou 50 minutes par semaine dans un horaire scolaire, mais plutôt dispensée sur des plages horaires plus longues, qui laissent le temps aux élèves de s'approprier les problématiques abordées et d'expérimenter par euxmêmes. En outre, ces auteurs prônent un enseignement des sciences intégré sans distinction entre les disciplines traditionnelles des facultés universitaires des sciences, telles biologie, physique, chimie, géologie, parce que les problèmes scientifiques ne sont jamais strictement disciplinaires. Enfin, l'école devrait prendre aussi en charge un enseignement technologique qui s'intéresse aux objets de la vie de tous les jours et qui fasse apparaître des liens entre les concepts scientifiques et la maîtrise technologique.

Rejoignant ces auteurs sur le postulat qu'une population d'élèves intéressés obtient de meilleurs résultats, nous devons accepter sa contraposée, c'est-à-dire le fait que les résultats moyens, que montrent entre autres les scores de PISA 2006, pourraient être dus en partie au manque de motivation des élèves. Mais alors si ce postulat est vérifié et si c'est l'organisation de l'enseignement des sciences qui n'encourage pas une attitude positive des élèves envers ces disciplines, celui-ci s'applique-t-il également au contexte de la Suisse romande? Qu'en est-il plus 
précisément de cette organisation dans les différents cantons, quelles en sont les similitudes et les différences? En se penchant sur les contenus prescrits, peut-on considérer que l'enseignement scientifique contribue à la formation d'une culture scientifique chez les élèves dans le sens défini par PISA? En effet, cette enquête ayant une légitimité de facto puisqu'elle teste les jeunes de 57 pays, dont tous les pays de l'OCDE, on peut s'interroger sur la correspondance entre les questions posées dans l'enquête pour tester la culture scientifique et les curriculums prescrits en Suisse romande.

Pour répondre à ces questions, il faut d'abord situer ce travail par rapport aux recherches sur les curriculums, puis définir les données analysées, enfin expliciter la méthodologie privilégiée. En reprenant les champs des thématiques de la recherche sur les curriculums définis par Crahay et al. (2006), notre réflexion se positionne dans le troisième axe, à savoir l'articulation entre les curriculums et les évaluations externes, mais plutôt que discuter leur éventuelle concurrence, nous tentons d'en mesurer leur plus ou moins grande concordance. A ce propos, il faut considérer que PISA, se démarquant d'autres enquêtes auprès des élèves qui se basent sur les plans d'études pour établir les contenus des tests, choisit comme objet pour ses questions des problématiques débattues dans la société et intéressant les jeunes (cf. annexe 2). Par la force des choses, ce travail touche aussi la dérangeante question de la relative réussite de l'école dans la formation scientifique des élèves et donc de l'efficacité des choix éducatifs, mais comme d'autres travaux, nous laissons cet aspect en arrière-plan. Quant au curriculums, nous retenons pour notre analyse, de façon plus restrictive que Landsheere (1991), d'une part l'organisation scolaire de tous les enseignements de l'école secondaire obligatoire considérés comme des sciences de la nature ou expérimentales, selon les nomenclatures cantonales et d'autre part la liste des contenus qui sont abordés dans ces cours, avec les intentions annoncées et les méthodes préconisées, mais à l'exclusion des manuels scolaires, de l'évaluation des apprentissages des élèves et des dispositions relatives à la formation des enseignants.

En ce qui concerne notre méthodologie, nous nous appuyons avant tout sur la description comparative des curriculums en lien avec notre question de recherche puisque, comme le souligne Reuter (2006), une méthode de recherche est «la forme prise par la démarche de travail pour répondre à une question» ( $\mathrm{p}$. 17). Nous avons ainsi, à partir du corpus de documents obtenus auprès des directions de la scolarité obligatoire secondaire des différents cantons ou via internet et du consortium romand PISA, construit nos données «en les sélectionnant et les catégorisant» (Reuter, 2006, p. 18). Ainsi, comme ce travail s'intéresse à l'enseignement des sciences au secondaire obligatoire dans les sept cantons romands en se plaçant au niveau de l'analyse de l'organisation scolaire et des curriculums officiels, dans une première étape nous établissons des tableaux comparatifs comportant les intitulés et la grille horaire de ces cours en fonction des différentes filières et sections scolaires ainsi que les durées hebdomadaires, ce qui permet d'évaluer l'importance relative au regard du temps d'enseignement total 
et les différences entre les cantons. En regardant ensuite de plus près de quel type de science il s'agit et à quels élèves ces cours sont proposés, nous brossons un tableau des axes principaux de l'enseignement scientifique suisse romand au secondaire obligatoire. Enfin, nous nous focalisons sur les contenus prescrits des cours de physique-chimie pour en relever les similitudes et les différences selon les cantons. Dans une deuxième étape, nous partons des questions de PISA 2006 portant sur les sciences et les organisons, autant que possible, en fonction de leur principale discipline de référence, biologie, physique, chimie, sciences de la Terre, astronomie, etc. Nous pouvons ainsi mettre en regard l'importance relative de ces différents domaines scientifiques du test avec les disciplines enseignées au secondaire obligatoire en Suisse romande. Plus finement, en ne retenant cette fois que les questions en lien avec la physique et la chimie, nous cherchons à identifier les sous-domaines testés et à éprouver leur concordance avec les contenus des curriculums de ces disciplines. Ces différentes correspondances nous permettent de poser de premières pistes de conclusion, d'une part à propos de la désaffection envers les sciences et d'autre part en ce qui concerne la distance entre curriculums prescrits et évaluations externes.

\section{Quel enseignement des sciences au secondaire I en Suisse romande?}

\section{La structure de L'enseignement secondaire obligatoire en Suisse romande}

Un enseignement des sciences est prévu dans tous les cantons romands au secondaire I. Cependant le secondaire I en Suisse romande ne recouvre pas les mêmes années scolaires dans les sept cantons concernés. En effet, si, pour la majorité des cantons romands, il concerne les élèves du $7^{\mathrm{e}}$ au $9^{\mathrm{e}}$ degré$^{7}$ - ce qui d'ailleurs ne correspond pas nécessairement à des jeunes exactement du même âge, l'école secondaire commence dans le canton de Neuchâtel en $\sigma^{\mathrm{e}}$ hétérogène, alors que, dans le canton de Vaud, c'est la $5^{\mathrm{e}}$ qui est une année d'orientation, mais qui appartient déjà au secondaire. D’autre part, le Valais, qui possède un Cycle d'Orientation (CO) de trois ans, voit l'effectif de sa dernière année fortement diminué, puisque les meilleurs élèves peuvent entrer au Lycée-collège déjà à la fin de leur $2^{\mathrm{e}}$ année du CO.

Les écoles de l'enseignement secondaire obligatoire n'ont pas non plus la même structure d'un canton à l'autre ${ }^{8}$. Si le Jura a adopté un système scolaire hétérogène avec, dès la $8^{\mathrm{e}}$, trois niveaux pour les disciplines français, mathématiques et allemand et quatre groupes d'options dont l'un renforcé en sciences, dans tous les autres cantons romands les élèves sont répartis en sections ou filières. Ainsi Berne connaît trois sections, Générale $(\mathrm{G})$, Moderne $(\mathrm{M})$ et une Section Prégymnasiale préparant aux Écoles de Maturité (SPEM ou P), de même que Fribourg où sont définies une filière à exigences de base (EB), une filière Géné- 
rale $(\mathrm{G})$ et une filière Prégymnasiale (P). À Genève ${ }^{9}$, il existe trois regroupements d'élèves en $7^{\mathrm{e}}$ année $A, B$ et $C$ qui ne sont plus que deux, $A$ et $B$ en $8^{e}$ et $9^{e}$. Neuchâtel a aussi trois sections, Maturité (Matu), Moderne (M) et Préprofessionnelle (P) et Vaud trois voies, l'une menant au baccalauréat (VSB), et les deux autres, générale (VSG) et voie à options (VSO), aux autres écoles secondaires ou à des apprentissages. Quant au Valais, la structure dépend des établissements pour les deux premières années, certains établissements du $\mathrm{CO}$ comportant des sections Générale $(\mathrm{G})$ et Secondaire $(S)$ et d'autres un système dit intégré, à niveaux I ou II en français, mathématiques et allemand et des cours hétérogènes pour les autres matières, la troisième année étant organisée partout selon le système intégré.

\section{L'organisation des sciences enseignées dans les diffé- rents cantons}

Ces différentes structures scolaires se répercutent sur toute une variété structurelle d'enseignements scientifiques. Dans le tableau que nous avons établi (annexe 1), adapté de celui de l'IRDP (2008), l'organisation des enseignements scientifiques au secondaire I est mise en évidence de façon synthétique avec le temps imparti dans la grille horaire aux différents cours. On en constate tout d'abord la complexité, puis la variété des dénominations. Si Neuchâtel s'aligne déjà sur le futur Plan d'études romand (PER) (CIIP, 2008) avec l'expression «sciences de la nature», les autres cantons ont des terminologies propres qui varient selon les filières et les degrés scolaires. Il peut donc y avoir des cours de «sciences», de «sciences expérimentales», de «sciences et techniques» ou de "physique ", de "physique et chimie» ou de «biologie», mais aussi des «TP de sciences», des «TP de biophysique» ou des «TP de biochimie». Ces particularités cantonales seront peut-être gommées avec l'avènement du PER, mais pour le moment il faut lire les plans d'études pour savoir ce qu'elles recouvrent exactement.

Le tableau en annexe 1 montre aussi le temps d'enseignement accordé aux sciences au secondaire I. Les cours de base de ces disciplines sont dispensées à un taux de une à deux périodes hebdomadaires par année scolaire ${ }^{10}$ dans tous les cantons. En revanche, les différences de temps accordé à ces disciplines pour des filières particulières peuvent être importantes, le cas extrême étant celui de Genève: au cours des trois ans de sa scolarité secondaire obligatoire, un élève genevois latiniste ne suivra que cinq périodes hebdomadaires de sciences (dont une seule de physique qui englobe aussi une initiation à la chimie), alors que son camarade qui a choisi le groupe 2 sciences se voit dispenser dix périodes de ces disciplines durant ces mêmes trois années. Si on prend de plus en compte le nombre de minutes d'une période, qui peut varier selon les cantons de 45 à 50 minutes, on constate que tous les élèves romands n'ont pas passé le même temps à étudier les sciences au cours de leur scolarité secondaire obligatoire. 


\section{Mais quelles sciences?}

Pour permettre la comparaison entre les enseignements scientifiques dans les différents cantons romands, au vu de la variété des dénominations, nous nous sommes tournée vers les contenus des Plans d'études (PE) cantonaux actuellement en vigueur, avant l'arrivée prévue dès la rentrée 2011 du PER. Tout en étant consciente du côté artificiel et subjectif que cela comporte, nous avons classé ces contenus en quatre grandes catégories: les sciences du vivant ou biologie (auparavant nommées sciences naturelles dans certains cantons); les sciences expérimentales, à l'exclusion du monde du vivant, physique et chimie; les sciences de la Terre, englobant des notions de géographie physique, de géologie et d'astronomie ${ }^{11}$; enfin, les cours de contenu non facilement attribuable à un de ces trois champs scientifiques. Ces catégorisations appellent un certain nombre de remarques. Tout d'abord, au contraire de la France par exemple où la formation scientifique se partage actuellement entre trois disciplines enseignées, les sciences physiques et chimiques, les sciences de la vie et de la Terre (SVT) et la technologie, nous n'avons trouvé que très peu de références ${ }^{12}$ aux sciences de la Terre dans les plans d'études romands de sciences. Il est vrai que certains sujets peuvent être traités en géographie, mais cela correspond peu à la tradition francophone où cette discipline est considérée comme une science humaine. Pour preuve, c'est bien dans les programmes de sciences qu'on trouvait dans la première moitié du $20^{\mathrm{e}}$ siècle la mention de l'étude du "règne minéral», remplacé ensuite par la "géologie» (voir par exemple le programme du collège inférieur genevois entre 1925 et 1950 dans les Plans d'études du Collège des années 1925 à 1953), mais ce sujet semble avoir disparu actuellement. Pour cette raison, nous avons renoncé à une catégorie sciences de la Terre.

De même, la quatrième catégorie nous a posé problème. En effet, nous avons choisi de ne pas y insérer d'office les cours appelés «TP» ou «laboratoire», car que ce soit à Berne, dans le Jura ou dans le canton de Vaud, les programmes de ces TP comportent explicitement une composante d'apprentissage des démarches expérimentales, mais tout aussi explicitement des objets de savoir de biologie ou de physique. C'est donc par exclusion, quand le sujet à traiter est laissé au choix de l'enseignant dans une palette d'activités qui relèvent de différentes disciplines de référence et dont l'objectif annoncé de placer l'élève dans une posture scientifique, que nous avons utilisé cette catégorie. Cependant, nous n'interprétons pas ces choix curriculaires comme l'idée que les sujets traités pourraient devenir, sinon des prétextes, du moins interchangeables pour enseigner une démarche scientifique qui serait indifférente au contexte et au problème étudié. A ce sujet, De Vecchi (2006) signale, à la suite des épistémologues contemporains des sciences comme Kuhn ou Hakking, que: «ce serait une erreur de croire qu'il existe une méthode scientifique générale, universelle, qui pourrait s'appliquer à tous les exemples de recherches.» (p. 46). Malgré ces réserves, nous avons finalement choisi de maintenir cette catégorie hybride en l'intitulant démarche scientifique / expérimentale parce qu'elle nous semble correspondre à l'objectif voulu par les 
concepteurs de programmes que les élèves expérimentent des situations variées dans le cadre des différentes disciplines scientifiques, où des démarches scientifiques spécifiques sont mises en jeu.

Une autre difficulté pour établir ces catégories est apparue à cause de l'intégration dans certains cours de sujets rattachés aussi bien à la physique-chimie qu'à la biologie, comme le cours Sciences (VS) ou les TP sciences (BE). Si cette intégration pourrait montrer que les curriculums romands vont déjà dans le sens d'un enseignement interdisciplinaire au secondaire I, il semble, en regardant de plus près les contenus, que l'interdisciplinarité n'est pas totalement réalisée, sauf peut-être en 1 ère année du CO valaisan avec des sujets comme l'air ou l'eau. Dans la majorité des autres cas, il s'agit plutôt de cohabitation dans le même cours de sujets appartenant aux différentes sciences. Pour notre décompte, nous sommes alors partie des indications du PE, et avons partagé le temps total prévu en fractions de temps pour la biologie ou la physique-chimie, en fonction du nombre et du type des sujets. Ce partage peut être la source d'un biais important dans nos données, un enseignant pouvant décider d'accorder la moitié de l'année scolaire à l'unique titre de biologie du PE (par exemple le corps humain et la santé en $2^{\mathrm{e}}$ année au $\mathrm{CO}$ valaisan) et de traiter pendant l'autre demi-année les quatre champs de physique et les quatre champs de chimie prévus au programme. Nous avons plutôt choisi d'attribuer un tiers temps à chaque discipline (noté dans le tableau 2 sous forme de fraction avant la durée hebdomadaire du cours $\left.^{13}\right)$.

Tableau 2: Temps des enseignements de sciences au secondaire I en Suisse romande

\begin{tabular}{|c|c|c|c|}
\hline & Sciences de la vie - biologie & Physique-chimie & $\begin{array}{l}\text { Démarche scientifique / } \\
\text { expérimentale }\end{array}$ \\
\hline Berne & $\begin{array}{l}\text { 7e } 90 \mathrm{~min} \\
\text { 7e fac. (TPS) } 90 \mathrm{~min} \\
\text { 8e } 45 \mathrm{~min} \\
8^{\text {e }} \text { opt. } 1 / 590 \mathrm{~min} \text { ou } \\
8^{\text {e }} \text { opt. (TP) } 9 / 1045 \mathrm{~min} \\
\text { 9e } 45 \mathrm{~min} \\
\text { 9e opt. (TP) } 9 / 1045 \mathrm{~min}\end{array}$ & $\begin{array}{l}8^{\mathrm{e}} 45 \mathrm{~min} \\
8^{\mathrm{e}} \text { opt. } 4 / 590 \mathrm{~min} \text { ou } \\
8^{\mathrm{e}} \text { opt. (TP) } 1 / 1045 \mathrm{~min} \\
9^{\mathrm{e}} 45 \mathrm{~min} \\
9^{\mathrm{e}} \text { opt. (TP) } 90 \mathrm{~min} \text { ou } \\
9^{\mathrm{e}} \text { opt. (TP) } 45 \mathrm{~min} \text { ou } \\
9^{\mathrm{e}} \text { opt. (TP) } 1 / 1045 \mathrm{~min}\end{array}$ & \\
\hline Total BE & 180 min à $324 \mathrm{~min}$ & 90 à $207 \mathrm{~min}$ & \\
\hline Fribourg & $\begin{array}{l}7^{\mathrm{e}} 100 \mathrm{~min} / 50 \mathrm{~min} \\
8^{\mathrm{e}} 100 \mathrm{~min}\end{array}$ & $9^{\mathrm{e}} 100 \mathrm{~min}$ & \\
\hline Total FR & 150 min à $200 \mathrm{~min}$ & $100 \mathrm{~min}$ & \\
\hline Genève & $\begin{array}{l}7^{\text {e }} 90 \mathrm{~min} \\
8^{\text {e }} 90 \mathrm{~min} \\
\text { 9e opt. } 90 \mathrm{~min}\end{array}$ & $\begin{array}{l}8^{\mathrm{e}} \text { opt. } 45 \mathrm{~min} \\
9^{\mathrm{e}} 45 \mathrm{~min} \\
9^{\mathrm{e}} \text { opt. } 90 \mathrm{~min}\end{array}$ & $\begin{array}{l}\text { 7e opt. } 45 \text { min } \text { (Obs. } \\
\text { scient.) }\end{array}$ \\
\hline Total GE & 180 min à $270 \mathrm{~min}$ & $45 \mathrm{~min}$ à $180 \mathrm{~min}$ & 0 min à $45 \mathrm{~min}$ \\
\hline Jura & $\begin{array}{l}\text { 7e } 4 / 590 \mathrm{~min} \\
\text { 7e opt. (TP) } 3 / 590 \mathrm{~min} \\
\text { 8e } 90 \mathrm{~min} \\
\text { 8e opt. } 3 / 490 \mathrm{~min} \\
\text { 9e } 90 \mathrm{~min}\end{array}$ & $\begin{array}{l}\text { 7e } 1 / 590 \mathrm{~min} \\
\text { 7e opt. (TP) } 2 / 590 \mathrm{~min} \\
\text { 8e } 90 \mathrm{~min} \\
\text { 8e opt. } 1 / 490 \mathrm{~min} \\
\text { 9e } 90 \mathrm{~min}\end{array}$ & \\
\hline Total JU & 252 min à 625 min & 198 min à $256 \mathrm{~min}$ & \\
\hline
\end{tabular}




\begin{tabular}{|c|c|c|c|}
\hline Neuchâtel & $\begin{array}{l}\text { 6e } 90 \text { min } \\
\text { 7e } 90 \text { min } \\
\text { 9e } 45 \text { min }\end{array}$ & $\begin{array}{l}8^{\text {e }} 90 \mathrm{~min} \\
9^{\text {e } 90 \mathrm{~min}} \\
\text { 9e opt. } 90 \mathrm{~min}\end{array}$ & \\
\hline Total NE & $225 \min$ & 180 min à 270 min & \\
\hline Valais & $\begin{array}{l}7^{\mathrm{e}} 1 / 2 \quad 100 \mathrm{~min} \\
8^{\mathrm{e}} 1 / 3 \quad 100 \mathrm{~min} \\
9^{\mathrm{e}} 1 / 3 \quad 100 \mathrm{~min}\end{array}$ & $\begin{array}{llll}7 \text { e } & 1 / 2 & 100 & \mathrm{~min} \\
8 \mathrm{e} & 2 / 3 & 100 \mathrm{~min} \\
9 \mathrm{e} & 2 / 3 & 100 \mathrm{~min}\end{array}$ & \\
\hline Total VS & $116 \mathrm{~min}$ & $182 \mathrm{~min}$ & \\
\hline Vaud & $\begin{array}{l}\text { 7e (TP) } 1 / 890 \mathrm{~min} \\
\text { (7e VSO } 1 / 845 \mathrm{~min}) \\
\text { 7e opt. } 1 / 2180 \mathrm{~min} \\
8^{\mathrm{e}} \text { (TP) } 1 / 390 \mathrm{~min} \\
8^{\mathrm{e}} \text { opt. } 1 / 2180 \mathrm{~min} \\
9^{\mathrm{e}}(\mathrm{TP}) 1 / 290 \mathrm{~min} \\
\left(9^{\mathrm{e}} \mathrm{VSO} 45 \mathrm{~min}\right) \\
9^{\mathrm{e}} \text { opt. } 1 / 2180 \mathrm{~min}\end{array}$ & $\begin{array}{l}\text { 7e (TP) } 3 / 890 \mathrm{~min} \\
(7 \mathrm{e} \text { VSO } 3 / 845 \mathrm{~min}) \\
8^{\mathrm{e}}(\mathrm{TP}) 2 / 390 \mathrm{~min} \\
9 \mathrm{e}(\mathrm{TP}) 1 / 290 \mathrm{~min} \\
\text { (9e VSO } 45 \mathrm{~min})\end{array}$ & $\begin{array}{l}5^{\mathrm{e}}(\mathrm{TP}) 45 \text { à } 135 \mathrm{~min} \\
6^{\mathrm{e}}(\mathrm{TP}) 45 \text { à } 135 \mathrm{~min} \\
7^{\mathrm{e}} 1 / 290 \mathrm{~min}\end{array}$ \\
\hline Total VD & $\begin{array}{l}86 \text { min à } 356 \text { min } \\
\text { (sans VSO) }\end{array}$ & 139 min (sans VSO) & $135 \mathrm{~min}$ à $315 \mathrm{~min}$ \\
\hline Total moyen & 170 min à 302 min & 133 min à 190 min & 81 min à 180 min \\
\hline \multicolumn{4}{|c|}{$\begin{array}{l}\text { Nota bene: le nombre d'années du secondaire obligatoire, le nombre de semaines d'école par année et de } \\
\text { minutes d'enseignement par semaine varie de façon importante d'un canton à l'autre, mais on peut } \\
\text { considérer que les élèves romands ont dans les majoritairement trois ans de secondaire obligatoire entre } \\
4000 \text { et } 5000 \text { minutes d'enseignement par semaine (IRDP, 2009), ce qui attribue aux sciences grosso } \\
\text { modo entre } 6 \% \text { et } 15 \% \text { du temps d'enseignement. } \\
\text { * la moyenne est obtenue en divisant par } 7 \text { le temps d'enseignement des sciences de la vie et de la physique-chi- } \\
\text { mie, mais par } 2 \text { celui de la troisième colonne puisqu'elle n'est présente que dans } 2 \text { cantons. }\end{array}$} \\
\hline
\end{tabular}

Si nous voulions maintenant établir le palmarès du canton qui prépare le mieux les élèves en sciences proportionnellement au temps scolaire accordé à ces disciplines, nous aurions ici des premiers éléments pour le faire, ce qu'il faudrait naturellement pondérer encore avec les pourcentages d'élèves dans les différentes filières scolaires cantonales. Rappelons que notre objectif est autre: dans cette première étape, en pointant les similitudes entre les dotations horaires cantonales, il s'agit plutôt de brosser un tableau général de l'enseignement des sciences au secondaire I en Suisse romande.

Notons tout d'abord, puisque ce tableau ne le montre pas, que les leçons de sciences portent dans la majorité des cas sur des plages de deux périodes, l'indication 45 minutes correspondant à un cours de 90 minutes dispensé pendant un semestre. Sans être des demi-journées complètes, cette durée permet tout de même la mise en place d'un travail expérimental à accomplir par les élèves. Ainsi la critique émise par Lechner (1994), expliquant le manque d'intérêt et les difficultés des élèves par les plages temporelles trop exigües et la rigidité de la structure scolaire, à savoir de trop brèves leçons hebdomadaires de 45 minutes et des leçons trop compartimentées entre les matières scientifiques, ne semble pas pleinement motivée à l'encontre de l'enseignement scientifique au secondaire I en Suisse romande. 
Relevons aussi (cf. annexe 1), pour prendre en compte l'injonction d'un enseignement des sciences intégré, que plusieurs cantons le privilégient, du moins dans les textes: Valais pour les trois ans, Neuchâtel pour les quatre, Vaud pour les élèves plus jeunes ( $5^{\mathrm{e}}$ et $6^{\mathrm{e}}$ année) et dans les filières non scientifiques, avec même un projet explicitement intitulé interdisciplinaire en VSG, Jura et partiellement Berne en $7^{\mathrm{e}}$ année (TP de sciences malheureusement facultatif).

Revenant aux totaux des durées hebdomadaires, malgré la grande imprécision du calcul, nous constatons en premier lieu la part considérablement plus importante consacrée à la biologie dans tous les cantons par rapport à la physique-chimie, sauf en Valais qui souffre plus que les autres du biais de notre découpage des cours intégrés en disciplines. En deuxième lieu, également dans tous les cantons, les sciences du vivant semblent être considérées comme plus accessibles à des élèves plus jeunes, puisqu'elles sont enseignées déjà en $7 \mathrm{e}$, alors que l'enseignement de la physique ne commence qu'en $8^{\mathrm{e}}$ et parfois seulement en $9^{\mathrm{e}}$ pour tous les élèves. Ce choix semble traditionnel: on retrouve par exemple à Genève au début $\mathrm{du} 20^{\mathrm{e}}$ siècle un enseignement de sciences naturelles dans les $7^{\mathrm{e}}$ et $6^{\mathrm{e}}$ classes du collège inférieur, correspondant aux actuels degrés $7^{\mathrm{e}}$ et $8^{\mathrm{e}} \mathrm{du} \mathrm{CO}$, remplacé dans la $5^{\mathrm{e}}$ classe (9e année) par l'enseignement de la physique et de la chimie (Plan d'études du collège, années 1925 à 1953).

En outre, la troisième colonne du tableau concernant la démarche est remplie pour deux cantons, Genève et Vaud, et dans les deux cas pour les élèves les plus jeunes ce qui incline à penser qu'il s'agirait plutôt d'une initiation aux sciences. Mais laissons le curriculum vaudois en définir les «compétences visées: pratiquer une approche de type scientifique à partir d'une situation faisant problème (aller-retour entre question, hypothèse, expérience, essai, erreur, débat scientifique, communication des observations)»(PEV 2006 Partie B, p. 13. 2) et, plus bas, "c'est un programme de sciences expérimentales, il n'y a pas lieu de séparer les aspects physique, biologique et chimique en les confiant à des maîtres différents». (PEV 2006 Partie B, p. 13. 2)

\section{Quels contenus en physique et chimie?}

Pour affiner notre analyse, nous avons étudié les contenus des PE de physique (et chimie) des différents cantons et avons pu mettre en évidence les principaux champs d'étude dans le tableau 3. Bien que ce tableau souffre de la simplification nécessaire à la synthèse des informations, qu'il ne prenne en compte que les curriculums prescrits et non la réalité du terrain et ses multiples contraintes, il confirme les constats déjà posés. On retrouve premièrement cette idée de démarche scientifique expérimentale comme champ d'étude cité explicitement sept fois dans les différents programmes cantonaux, en particulier dans le PE vaudois qui insiste pour chaque degré scolaire sur la nécessité de mettre les élèves en situation d'apprentissage des méthodes scientifiques. Deuxièmement, c'est encore le PE vaudois qui est le seul à faire référence à un enseignement de la technologie, en donnant l'exemple précis du panneau solaire thermique, mais en laissant toute li- 
berté aux enseignants d'imaginer d'autres objets techniques à étudier. Cet aspect de l'enseignement des sciences est dans la même ligne que l'enseignement de la technologie introduit dans les curriculums scientifiques en France et réclamé par Pfenning et al. (2002) en Allemagne. Notre constat est qu'il reste anecdotique dans les curriculums romands. Troisièmement, le PE bernois fait mention de l'écologie et le PE valaisan de l'environnement, indications qui peuvent être interprétées comme la volonté que l'enseignement scientifique soit en prise directe avec des problématiques interdisciplinaires de la vie réelle. Globalement enfin, tous ces PE sont bien éloignés de textes proposant un enseignement des sciences poussiéreux, sans lien avec les préoccupations sociétales actuelles et compartimenté dans des disciplines étanches les unes par rapport aux autres, qui attirent les foudres des experts.

Tableau 3: Sujets principaux abordés dans les enseignements de physique et chimie au secondaire I en Suisse romande

\begin{tabular}{|l|l|l|l|l|}
\hline Sujets & $\left(5^{\mathrm{e}}-6^{\mathrm{e}}\right) 7^{\mathrm{e}}$ & $8^{\mathrm{e}}$ & $9^{\mathrm{e}}$ & total \\
\hline Acoustique & & $\mathrm{BE}$ & & 1 fois \\
\hline Air - eau & NE; VS & & & 2 fois \\
\hline Astronomie & VD & & BE; JU & 1 fois \\
\hline Chaleur & VD & & VD; GE; FR & 3 fois \\
\hline Chimie & VD & BE; JU; NE; VS & 8 fois \\
\hline Électricité & VD & VS & BE; FR; GE; JU; NE; VD; VS & 9 fois \\
\hline Énergie & VD & VD & BE; NE; VD & 5 fois \\
\hline Magnétisme & VD & & & 1 fois \\
\hline Matière & JU; VD & BE; JU; NE; VS; VD & FR; GE; VD & 10 fois \\
\hline Mécanique & VD & BE; JU; VS & BE; FR; JU; NE; VD & 10 fois \\
\hline Optique & VD & BE; GE; JU; NE; VD & FR; VS & 8 fois \\
\hline Technologie & VD & & & 1 fois \\
\hline $\begin{array}{l}\text { Démarche scienti- GE; VD } \\
\text { fique expérimentale }\end{array}$ & BE; NE; VD & NE; VD & 7 fois \\
\hline
\end{tabular}

Dans cette liste, bien que les mêmes intitulés puissent recouvrir des contenus différents ou que les mêmes contenus puissent correspondre à des titres différents (par exemple le chapitre chaleur peut être intégré dans le domaine énergie, ou bien les changements d'états physiques peuvent être traités dans le chapitre chaleur, aussi bien que dans le chapitre matière), on relève la prééminence du domaine matière (modèle moléculaire et structure de la matière, états physiques et changements d'états). Les notions de mécanique comme la masse, les forces et les leviers, et parfois des concepts de cinématique comme la vitesse sont aussi présents dans les plans d'études de tous les cantons, sauf à Genève où ce chapitre est l'un des sujets à choix des enseignants pour le cours optionnel. Électricité (courant continu et circuits électriques) et optique sont aussi traitées dans tous les cantons, sauf à Genève qui les réserve au cours optionnel, mais de façon obligatoire. 
En conclusion provisoire, retenons à partir de ce travail d'analyse des curriculums romands de sciences que l'enseignement de la physique et de la chimie au secondaire obligatoire est plus tardif et moins doté en heures que celui des sciences de la vie. En outre, les sujets abordés sont à peu de choses près les mêmes dans les sept cantons. Ainsi, la structure de la matière, la mécanique (forces), l'optique et l'électricité se taillent la part du lion dans tous les cantons. Pour ces deux derniers sujets surtout, la possibilité de modéliser sans devoir recourir à un formalisme ou à une mathématisation poussée, la relative facilité de vérification des modèles à l'aide d'un travail expérimental, l'accessibilité et le faible coût du matériel jouent certainement un rôle dans ces choix.

\section{Une tentative d'analyse des contenus de l'en- quête PISA 2006 sciences}

\section{Les domaines de connaissances testés}

Dans le test PISA 2006, trente-sept «unités» concernent les sciences. Sans revenir sur la façon dont ces unités ont été conçues, testées, puis distribuées dans treize cahiers partiellement différents pour tester plus de questions que celles auxquelles pourrait répondre un élève dans le temps imparti, relevons que chaque unité, qui occupe entre une et trois pages du cahier, est constituée par un titre, un "stimulus», c'est-à-dire un texte éventuellement accompagné d'un tableau ou d'un graphique, d'un diagramme ou d'une illustration, correspondant à un contexte donné et de plusieurs questions (entre deux et six), que nous nommons items. Pour éviter le reproche fait aux précédents cycles PISA de tester davantage la capacité des élèves à lire et comprendre un texte que leurs compétences en sciences ${ }^{14}$, les textes sont relativement brefs (en moyenne une dizaine de lignes) et rédigés de façon aussi simple que possible. Parfois, un nouveau texte ou un graphique supplémentaire complète l'information nécessaire pour les items ultérieurs. Le stimulus permet de contextualiser les items et de poser les questions «à propos» des sciences. Toute question demandant une explication scientifique ou faisant appel à une démarche scientifique selon la terminologie PISA impose en effet des informations circonstanciées.

D'autres dimensions ont été prises en compte toutefois pour construire cette batterie de questions, et ce de façon prioritaire par rapport aux quatre systèmes scientifiques concernant les items « en " sciences et à la catégorisation "démarche scientifique» versus «explications scientifiques» des items «à propos» des sciences. Il est important de le préciser ici, parce que ce sont ces dimensions qui orientent fortement le contenu des questions, les systèmes scientifiques restant en quelque sorte en arrière plan. Ainsi, les questions sont choisies dans cinq champs d'application (voir annexe 2) présents dans les débats médiatisés dans notre société contemporaine, afin de remplir les critères suivants: les connaissances retenues doivent être "pertinentes par rapport à des situations de la vie réelle», «représen- 
ter des concepts fondamentaux d'une utilité durable» et «être en adéquation avec le niveau de développement des jeunes de 15 ans».

Sachant cela, nous avons repris l'ensemble des 108 items des 37 unités et avons tenté d'appliquer la même catégorisation, aussi arbitraire qu'elle soit, que celle que nous avons choisie pour les curriculums romands. Pour ce faire, sans nous baser sur les champs de l'annexe 2, ni l'analyse des huit unités publiques du rapport PISA 2006 (OCDE, 2007, p. 50), nous avons d'abord attribué les items aux domaines scientifiques concernés quand cela était possible. Cela nous a permis de constater aussitôt que plusieurs unités font appel à plus d'un système scientifique. Comme cela était prévisible à partir de la description des dimensions et contextes de l'annexe 2 (par exemple la dimension santé recouvre biodiversité, qualité de la vie humaine, aliments, etc. tous sujets en lien avec la biologie), le tableau 4 met en évidence la prépondérance des unités et des items reliés aux sciences du vivant. A noter que le total dépasse 37, car plusieurs d'entre-elles font appel à deux ou plusieurs systèmes.

Tableau 4: Décompte des unités et des items selon les systèmes ou disciplines concernés

\begin{tabular}{|l|l|l|}
\hline Champ disciplinaire & Nombre d'unités & Nombre d'items \\
\hline Biologie - sciences de la vie & 18 & 50 \\
\hline Physique & 14 & 17 \\
\hline Chimie & 8 & 11 \\
\hline Sciences de la Terre - astronomie & 9 & 22 \\
\hline Technologie & 3 & 8 \\
\hline Total & 52 (supérieur à 37) & 108 \\
\hline
\end{tabular}

L'attribution des unités et des items aux disciplines scolaires a été difficile, et nous nous sommes quelques fois distancée des choix du rapport PISA, entre autres parce que les questions «à propos» des sciences ne sont pas catégorisées par PISA selon l'un des quatre systèmes, comme si, puisqu'il s'agit d'une explication de type scientifique, la discipline de référence n'était pas importante. Par exemple, nous considérons l'unité "écrans solaires» (OCDE, 2007, p. 90) portant sur la mesure de la capacité des crèmes solaires à arrêter les rayons UV comme relevant des systèmes physiques, sous catégorie chimie, car elle s'appuie sur les effets de la lumière sur du papier photosensible. Le rapport PISA l'attribue à la dimension santé et relevant du contexte personnel, sans attribution à un domaine scientifique particulier (cf. annexe 2). Nous rejoignons en revanche l'analyse PISA pour estimer que les items de cette unité font appel à une démarche scientifique. 
La première conclusion à tirer de cette catégorisation est la confirmation de la correspondance entre les sujets testés et l'enseignement des sciences en Suisse romande, à l'exception des sciences de la Terre: la majorité des items PISA concernent la discipline scientifique la plus étudiée au secondaire I, les sciences de la vie, et très peu de questions relèvent de la technologie qui n'est pas enseignée en tant que telle en Suisse romande. Le fait que certaines unités aient relativement peu d'items, par exemple en chimie ou en physique, s'explique par des questions plus pointues dans ces domaines, alors que des unités d'autres systèmes permettent de poser beaucoup de questions, mais souvent plutôt «à propos» des sciences. Ceci fait l'objet de notre analyse suivante.

\section{Les types de connaissances testées}

Est-ce que les jeunes qui n'ont pas étudié les sciences de la Terre ou la technologie sont vraiment handicapés pour répondre aux questions du test? Est-ce que cela remet en cause leur culture scientifique, au sens de PISA? Nous distançant d'autres études qui repartent des scores PISA par analyser a posteriori les compétences des élèves en fonction des types de questions posées (voir par exemple Coppens, 2009), pour traiter de cette problématique nous faisons une analyse $a$ priori simplifiée des items en fonction de la nécessité de disposer de connaissances «en» ou «à propos» des sciences, pour reprendre la terminologie PISA.

Tableau 5: Distinction entre connaissances "en" et "à propos» des sciences, démarche de type scientifique et liens entre les informations du stimulus

\begin{tabular}{|l|c|c|}
\hline Discipline concernée & $\begin{array}{l}\text { Items faisant appel à des connais- } \\
\text { sances «en» sciences non présentes } \\
\text { dans le stimulus }\end{array}$ & $\begin{array}{l}\text { stems ne faisant pas appel à des connais- } \\
\text { sances «s sciences autres que celles données } \\
\text { des sciences) }\end{array}$ \\
\hline $\begin{array}{l}\text { Biologie - sciences } \\
\text { de la vie }\end{array}$ & 21 & 29 \\
\hline Physique & 9 & 3 \\
\hline Chimie & 13 & 10 \\
\hline $\begin{array}{l}\text { Sciences de la Terre et } \\
\text { astronomie }\end{array}$ & 0 & 8 \\
\hline Technologie & 52 & 56 \\
\hline Total & & 6 \\
\hline
\end{tabular}

Le tableau 5 permet de constater l'équilibre, à quatre unités près, entre les items requérant des connaissances "en» sciences et ceux dont la réponse peut être trouvée par un raisonnement à partir du stimulus. Parmi ces items «à propos» des sciences, certains font clairement appel à des démarches de type scientifique, telles le choix d'une investigation de type scientifique à partir des propriétés des 
données, la distinction entre un fait et une hypothèse, la prise en compte des caractéristiques des mesures, la vigilance d'un contrôle d'autres facteurs pouvant influencer les résultats, alors que les autres demandent principalement la capacité de mettre en correspondance les informations du stimulus, compétence très importante en sciences mais aussi dans d'autres domaines de la connaissance.

Le tableau montre que c'est dans les sciences de la vie qu'on retrouve le plus d'items faisant appel au raisonnement, relativement au nombre d'items qui nécessitent des connaissances de la discipline. Cet état de fait n’a pas manqué de nous étonner, puisque nous nous attendions au contraire à trouver plus de questions de connaissances en biologie. En effet, comme la biologie est très présente dans les préoccupations sociétales à travers la problématique de la santé, l'enquête PISA aurait pu davantage miser dans ce domaine sur des connaissances faisant partie de la culture scientifique. Cet équilibre est inversé en physique et chimie, alors qu'il est maintenu en sciences de la Terre, mais les connaissances nécessaires pour répondre aux items relatifs à ce dernier champ sont rarement pointues. Enfin pour les items en lien avec la technologie, une réflexion plus ou moins scientifiquement construite semble suffire pour donner la bonne réponse, ce qui évite de désavantager les élèves qui n’ont pas étudié cette matière en classe.

\section{Les connaissances testées en physique et chimie}

Pour conclure cette revue de l'enquête PISA sciences, nous portons un regard sur tous les items concernant, selon nous, la physique et la chimie pour les mettre en relation avec les sujets traités dans les curriculums romands. Le tableau 6 rappelle le champ des connaissances retenu par PISA pour définir les systèmes physiques.

Tableau 6: les champs de connaissances des systèmes physiques du cycle PISA 2006 (OCDE, 2007, p. 43)

\section{Systèmes physiques}

- Structure de la matière (modèles de particules et liaisons intramoléculaires)

- Propriétés de la matière (changements d'état et conductivité thermique et électrique)

- Changements chimiques de la matière (réactions, transfert d'énergie et acides et bases)

- Forces et mouvements (vitesse et friction)

- Énergie et transformation de l'énergie (conservation, dissipation et réactions chimiques)

- Interactions entre l'énergie et la matière (ondes lumineuses et radioélectriques et ondes sonores et sismiques)

La correspondance entre ces contenus et ceux des PE romands (voir tableau 3) est immédiatement apparente. Peut-être tous les élèves romands n'ont-ils pas ap- 
profondi certaines propriétés de la matière, comme la conductivité électrique, qui est probablement étudiée dans le chapitre électricité, ou peut-être les concepts de transfert d'énergie lors des réactions chimiques vont-ils au-delà des initiations à la chimie dispensées dans les cours de physique du secondaire obligatoire, mais globalement le choix des contenus est en accord.

En outre, la traduction concrète de ces têtes de chapitres en questions PISA confirme, en ce qui concerne les systèmes physiques, la correspondance entre les curriculums et les sujets testés dans l'enquête internationale. En effet, nous dénombrons trois items sur les états de la matière, ainsi que six items sur les sujets suivants: conduction de la chaleur (chaleur), masse volumique (matière), constitution microscopique de la matière (matière), mesure de la conductivité (électricité), production d'énergie (énergie) et propriétés des aimants (magnétisme), ce dernier sujet étant prévu seulement dans le curriculum vaudois. Les neuf items de connaissances en chimie, quant à eux, concernent essentiellement des réactions chimiques, par exemple la différence entre une réaction chimique et un changement d'état physique, les conditions des réactions d'oxydation, ou le sens d'une formule chimique, ce qui recouvre assez bien l'initiation à la chimie du secondaire obligatoire en Suisse romande.

\section{Conclusion}

Au terme de cette analyse de l'enseignement romand du secondaire obligatoire en sciences et en particulier des contenus des curriculums romands de physiquechimie et de l'étude des questions de l'enquête PISA 2006 sur les sciences, nous pouvons tirer un certain nombre de conclusions. Tout d'abord, selon les textes, aussi bien l'organisation de l'enseignement que les curriculums romands vont déjà dans le sens des injonctions des experts inquiets de la désaffection des jeunes à l'égard des sciences. On trouve en effet un enseignement généralement dispensé en plages de 90 ou 100 minutes, avec un accent mis sur la possibilité pour les élèves d'expérimenter par eux-mêmes dans les nombreux TP ou cours-laboratoires proposés. Même si la mise sur pied de cours plus interdisciplinaires est sans doute encore insuffisante, la tendance existe d'après les intitulés des cours. Parallèlement un accent est mis sur le développement d'une posture scientifique chez les élèves tout autant que sur l'apprentissage de notions scientifiques. Pour ce dernier aspect, il y a concordance entre ces curriculums prescrits et ce qui est testé par l'enquête PISA sciences 2006 avec l'équilibre maintenu entre items «en» sciences et items «à propos» des sciences.

Le même constat de concordance peut se faire à propos des contenus disciplinaires enseignés en Suisse romande et les domaines abordés par les questions PISA, hormis l'absence d'un enseignement des sciences de la Terre et de trop faibles tentatives d'introduire l'étude d'objets techniques. Enfin, en ce qui concerne l'enseignement de la physique et de la chimie, la même proximité existe entre ce 
qu'il est prévu d'être enseigné et les domaines testés par l'enquête PISA.

Cependant ces plans d'études cantonaux sont appelés à disparaître à partir de la prochaine rentrée scolaire pour être remplacés par le PER. Est-ce que celui-ci va être en cohérence avec PISA dans le domaine des sciences? Il est frappant, en tout premier lieu, de lire dans le PER que

La formation scientifique de l'élève a pour but de lui donner les outils et repères nécessaires à une action citoyenne dans une société où le débat intègre fréquemment des dimensions scientifiques. (CIIP, 2008, p. 287)

Ceci est à peu de choses près le même argument donné par PISA pour expliquer l'importance d'une culture scientifique chez tous les élèves. Deuxièmement le poids et le temps plus important attribué aux sciences de la vie, ainsi que, selon les cantons, l'anticipation de cet enseignement par rapport à la physique-chimie seront probablement maintenus: deux objectifs d'apprentissage concernent la biologie, alors que seul le MSN36 «analyser des phénomènes naturels et des technologies à l'aide de démarches caractéristiques des sciences expérimentales» fait référence aux deux autres disciplines. Si un enseignement de la technologie pointe dans cet objectif et sa composante «en utilisant un modèle pour expliquer et/ou prévoir un phénomène naturel ou le fonctionnement d'un objet technique», une approche des sciences de la Terre n'est pas prévue dans le domaine Mathématiques et Sciences de la nature. En effet, les champs d'étude retenus sont Matière, Optique, Mécanique, Électricité, Énergie, avec comme arguments la nécessité d'une base scientifique pour percevoir correctement les phénomènes de la vie quotidienne, l'occasion donnée par ces thèmes de démonter des représentations intuitives fausses, la possibilité de faire jouer des modèles microscopiques pour expliquer le macroscopique, l'appel, fait par deux de ces champs, au concept de conservation, essentiel en sciences, et les liens possibles avec d'autres domaines ou disciplines «notamment la dynamique du climat, les dangers de l'électricité, le développement durable». (CIIP, 2008, p. 287)

Le PER insiste aussi sur l'importance de la démarche: «Les Objectifs d'aprentissage de Sciences de la Nature doivent mettre l'élève en situation de démarche expérimentale aussi souvent que possible, car la science est autant un processus qu'une liste de connaissances à acquérir.» (CIIP, 2008, p. 287). Si PISA 2006 sous forme de test papier crayon n'a pas pu éprouver les compétences des élèves à adopter concrètement une démarche expérimentale, les items «à propos» des sciences poursuivaient ce même but.

En conclusion, un consensus existe entre ce que l'élève devrait savoir pour détenir une culture scientifique et les buts, présents ou prévus dans un futur proche, visés par l'école romande dans l'enseignement des sciences. L'organisation scolaire romande a déjà mis en application une partie des recommandations des experts visant à renforcer l'intérêt des jeunes vis-à-vis des sciences. Ce n'était pas notre objet d'estimer si les élèves sont sensibilisés assez tôt aux sciences à l'école 
primaire, mais nous ne saurions croire que tout le problème se trouve là. Au contraire, cette désaffection à l'égard des sciences est d'autant plus grave qu'il est difficile d'en identifier les causes, même si un certain nombre d'entre elles ont été avancées. Un levier se trouve peut-être dans la formation des enseignants qu'il s'agirait de rendre conscients de l'importante tâche qui leur incombe pour la société de demain: rendre les sciences plus attrayantes pour la majorité des élèves.

\section{Bibliographie}

CDIP. (2007). PISA 2006: Les compétences en sciences et leur rôle dans la vie. Rapport national. Neuchâtel: OFS.

CDIP. (2009). PISA 2006: Études sur les compétences en sciences. Rôle de l'enseignement, facteurs déterminant les choix professionnels, comparaison de modèles de compétences. Neuchâtel: OFS.

CIIP. (2008). Plan d'études romand (PER) [version électronique]. Consulté en décembre 2009 dans http://www.ciip.ch/index.php

Conseil fédéral (2010). Pénurie de spécialistes MINT en Suisse. Ampleur et causes de la pénurie de personnel qualifié dans les domaines MINT (mathématiques, informatique, sciences naturelles et technique). Août 2010. Consulté en novembre 2010 dans http://www.sbf.admin.ch/htm/dokumentation/publikationen/uni/Bericht_Fachkraeftemangel_fr.pdf

Coppens, N. (2009). La culture scientifique des élèves français de quinze ans. Résultats de l'évaluation internationale PISA 2006. Bulletin de l'union des professeurs de physique et de chimie, 910, (1), 41-52.

Coradi, M., Denzler, S., Grossenbacher, S. \& Vanhooydonck, S. (2003). Les maths et les sciences n'ont-elles plus la cote? Rendre l'enseignement des mathématiques, des sciences et des branches techniques plus attractif et assurer un traitement équitable aux filles et aux garçons. (Rapport de tendance CSRE No 6). Aarau: CRSE.

Crahay, M., Audigier, F. \& Dolz, J. (2006). Introduction. En quoi les curriculums peuvent-ils être objets d'investigations scientifiques? In F. Audigier, M. Crahay \& J. Dolz (Éd.), Curriculum, enseignement et pilotage (pp. 7-37). Bruxelles: De Boeck.

De Vecchi, G. (2006). Enseigner l'expérimental en classe: Pour une véritable éducation scientifique. Paris: Hachette éducation.

Durner, H. (2002). Gymnasiale Bildung im Spannungsfeld von Allgemeinbildung und Studierfähigkeit. In U. Herrmann, Naturwissenschaften - Gymnasium - Universität (pp. 2835). Ulm: Universitätsverlag.

Farquet, R. (1993). Le tremplin et l'obstacle. L'insertion des disciplines d'enseignement au collège de Genève (Tome 1). Genève: DIP.

Giordan, A. \& De Vecchi, G. (1994). L'enseignement scientifique. Comment faire pour que ça marche? Nice: Z'éditions.

IRDP. (2008). Grilles-horaires officielles. Enseignement primaire et secondaire premier cycle. Suisse Romande et Tessin. Tableaux comparatifs. Année scolaire 2008-2009 [version électronique]. Consulté en octobre 2008 dans http://www.irdp.ch/publicat/irdp_dossiers_comparatifs/grillesecoles/grillesecolesdocumentcomplet0809.pdf

IRDP. (2009). Temps scolaire effectif des élèves. Éducation préscolaire. Enseignement primaire et secondaire premier cycle. Suisse Romande et Tessin. Tableaux comparatifs. Année scolaire 20092010 [version électronique]. Consulté en novembre 2010 dans http://www.irdp.ch/publicat/irdp_dossiers_comparatifs/tempsscolaire/2009_2010/tempsscolairedocumentcomplet0910.pdf

Kuhn, T. S. (1983, [1962]). La structure des révolutions scientifiques. Paris: Flammarion 
(Champs).

Labudde, P. (2000). Konstruktivismus im Physikunterricht der Sekundarstufe II. Bern: Haupt Verlag.

Landsheer, G. (1991). Dictionnaire de psychologie. Coordonnée par R. Dorot \& F. Parot. Paris: PUF.

Lechner, H. (1994). Warum hat physikalische und chemische Bildung zu wenig Akzeptanz bei den Schülern? Pädagogik und Schulalltag, 49, (3), 364-371.

Nidegger, Ch. (Éd.) (2008). PISA 2006: Compétences des jeunes romands. Résultats de la troisième enquête PISA auprès des élèves de $9^{e}$ année. Neuchâtel: IRDP.

OCDE. (2007). PISA 2006: Les compétences en sciences, un atout pour réussir. Volume 1 - Analyse des résultats. Paris: OCDE.

Perrenoud, Ph. (1993). Curriculum: Le formel, le réel, le caché. In J. Houssaye (Éd.), La pédagogie: Une encyclopédie pour aujourd'hui (pp. 61-76). Paris: ESF.

Pfenning, U., Renn, O. \& Mack, U. (2002). Zur Zukunft technischer und naturwissenschaftlicher Berufe. Strategien gegen den Nachwuchsmangel. Studie im Auftrag der Akademie für Technikfolgenabschätzung in Baden-Württemberg [version électronique]. Consulté en janvier 2010 dans http://elib.uni-stuttgart.de/opus/volltexte/2004/1823/pdf/studieIngmangel.pdf

Raulin, D. (2008). Le socle commun des connaissances et compétences. Paris: Hachette.

Reuter, Y. (2006). Penser les méthodes de recherche en didactique(s). In M.-J. Perrin-Glorian \& Y. Reuter (Éd.), Les méthodes de recherche en didactique (pp. 13-26). Villeneuve d'Ascq: Presses Universitaires du Septentrion.

Rocard, M., Csermely, P., Jorde, D., Lenzen, D., Walberg-Henriksson, H. \& Hemmo, V. (2007). Science education now. A renewed pedagoy for the future of europe. Bruxelles: European Commission.

http://ec.europa.eu/research/science-society/document_library/pdf_06/report-rocard-onscience-education_en.pdf Consulté en décembre 2010.

Zwick, M. M. \& Renn, O. (2000). Die Attraktivität von technischen und ingenieurwissenschaftlichen Fächern bei der Studien- und Berufswahl junger Frauen und Männer. Stuttgart: Akademie für Technikfolgenabschätzung in Baden-Württemberg [version électronique]. Consulté en janvier 2010 dans http://elib.uni-stuttgart.de/opus/volltexte/2004/1768/ pdf/berufswahl.pdf

\section{Plans d'études consultés}

Berne: Plan d'études pour les écoles secondaires de langue française. Sciences naturelles (Biologie / Chimie / Physique / Travaux pratiques. Editions scolaires du canton de Berne. 1996.

Fribourg: Direction de l'instruction publique, de la culture et du sport. Service de l'enseignement obligatoire de langue française Plan d'études Sciences, $7^{\mathrm{e}} 8^{\mathrm{e}}$ et $9^{\mathrm{e}}$ années. Cycle d'orientation du canton de Fribourg. Juin 2006.

Genève: Département de l'instruction publique. Cycle d'orientation de l'enseignement secondaire. Plan d'études Physique-chimie. Genève 2001.

Jura : République et canton du Jura. Plan d'études. Ecole secondaire. Sciences expérimentales. Juillet 2007.

Neuchâtel: DECS. Service de l'enseignement obligatoire. PENSE. Plan d'études du degré 6. Plan d'études du secondaire 1. Degrés 7-8-9. Mars 2006 et 2007.

Valais: Département de l'éducation, de la culture et du sport. Sciences. Objectifs généraux et spécifiques. Degrés 7-9. Juin 2003.

Vaud: DFJ- Direction générale de l'enseignement obligatoire. Plan d'études vaudois. Version 2007. PER: http://www.plandetude.ch/web/guest/systemic?domainId=56\&\&courseId=240\&rvn=3. Consulté en novembre 2010. 


\section{Notes}

1 Conseil national, Postulats $n^{\circ}$ 05.3508 Fetz «Accroître le pourcentage des femmes dans les cursus de mathématiques, sciences naturelles et disciplines techniques», $\mathrm{n}^{\circ} 07.3538$ Hochreutener «Formations en sciences naturelles et techniques», 07.3747 Recordon «Déficit de la Suisse dans les professions scientifiques», $\mathrm{n}^{\circ}$ 07.3810 Widmer «Il faut plus d'étudiants en ingénierie et en sciences naturelles», $\mathrm{n}^{\circ} 09.3930$ Kiener Nellen "Égalité des sexes. Davantage de femmes dans les professions techniques, les filières mathématiques et les sciences naturelles».

2 Conseil national, Postulat n ${ }^{\circ} 00.3697$ Riklin «Renforcer l'intérêt pour l'étude des branches scientifiques".

3 Conseil national, Postulat $n^{\circ}$ 98.3078 Stump «Les mathématiques sont aussi l'affaire des femmes».

4 Programme International pour le Suivi des Acquis des Élèves, série de tests passés tous les trois ans à partir de 2000 par un échantillon d'élèves de 15 ans dans tous les pays de l'OCDE et d'autres en littératie, mathématiques et sciences. Il s'agit ici des résultats de PISA 2000.

5 Third International Mathematics and Science Study lancée par l'IEA (Association internationale pour l'évaluation du rendement scolaire). 45 pays ont participé à cette étude qui concernait les élèves des degrés scolaires 6, 7 et 8 ainsi que 10 et 12 en 1995.

6 Le rapport prend en compte des facteurs internes aux personnes, externes à celles-ci et des combinaisons des facteurs internes et externes.

7 Dans ce texte, nous utilisons encore l'ancienne dénomination des degrés scolaires. Avec l'entrée en vigueur de HarmoS et l'obligation de scolariser tous les enfants à partir de 4 ans, ces classes deviendront les degrés scolaires 9 à 11 .

8 Les différents systèmes du secondaire I romand sont décrits dans Nidegger (2008).

9 Genève possède trois établissements du $\mathrm{CO}$ (sur vingt) dits hétérogènes où les élèves sont répartis selon des niveaux en mathématiques et allemand et des options. En ce qui concerne les sciences, à part un cours obligatoire pour tous les élèves pendant un semestre en $9^{\mathrm{e}}$ année, elles sont données en cours à option dans les vingt établissements du canton, définissant ainsi une filière scientifique dans les deux regroupements $\mathrm{A}$ et $\mathrm{B}$.

10 Certains de ces enseignements sont donnés au taux de deux périodes hebdomadaires sur un semestre.

11 L'astronomie peut aussi faire partie de l'enseignement de la physique, souvent comme cours optionnel.

12 A Berne francophone, dans les TP sciences en 7 e année, il y est fait mention de la géologie comme discipline de référence: ainsi il y est par exemple proposé une initiation à la spéléologie avec la visite d'une grotte touristique ou des observations de fossiles, mais ces sujets restent marginaux.

13 Les indications temporelles de ce tableau sont à considérer comme uniquement indicatives, elles peuvent, selon les cantons, varier fortement d'un établissement à un autre et d'un enseignant à un autre.

14 Quelques questions portant sur les sciences ont été testées dans les précédents cycles PISA en 2000 (litératie) et 2003 (culture mathématique).

\footnotetext{
Mots clés: Curriculums, PISA sciences, secondaire obligatoire, Suisse romande
} 


\section{Naturwissenschaftlicher Unterricht in der verpflichtenden Schule der welschen Schweiz im Vergleich mit der naturwissenschaftlichen Grundbildung von Jugendlichen}

Zusammenfassung

PISA 2006 hat die naturwissenschaftliche Grundbildung ("scientific literacy") von Jugendlichen in 57 Ländern untersucht. In einer Zeit, in der alle Länder der OECD zunehmendes Desinteresse sowie i.a. unbefriedigende Kompentenzen von Jugendlichen im Bereich Naturwissenschaften feststellen, ist es wesentlich den Beitrag der Schule, und insbesondere der verpflichtenden Schule, zu dieser naturwissenschaftlichen Grundbildung einzuschätzen. Dieser Beitrag liefert eine Zusammenstellung der naturwissenschaftlichen Curricula in der französischsprachigen Schweiz (Romandie) mit dem Ziel, diese Unterrrichtsvorgaben sowohl den Experten-Empfehlungen zur Steigerung des naturwissenschaftlichen Interesses im Vergleich mit den PISA-Fragen im Bereich Naturwissenschaften gegenüberzustellen. So wird eine Darstellung der naturwissenschaftlichen Lehre in den sieben französischsprachigen Kantonen der Schweiz gegeben.

Schlagworte: Curriculum, PISA-Naturwissenschaften, Sekundarstufe I, französisch-sprachige Schweiz

\section{L'insegnamento delle scienze nelle scuole medie della Svizzera romanda alla luce delle inchieste internazionali sulla cultura scientifica dei giovani}

Riassunto

PISA 2006 ha misurato la cultura scientifica ("scientific literacy") dei giovani in 57 paesi. A un'epoca dove tutti i paesi dell'OEDC sono confrontati al disinteresse verso le scienze dei giovani, che fanno inoltre prova di competenze generalmente insoddisfacenti in questo campo, è cruciale interrogarsi sul contributo della scuola, e in particolare dell'insegnamento d'obbligo a questa cultura. In questo articolo vengono analizzati i vari curricula della scuola media alla luce delle domande PISA mettendo così in perspettiva l'insegnamento scientifico e le ingiunzioni degli esperti per rinforzare un'attitudine positiva verso le scienze. Questo lavoro presenta un quadro dell'insegnamento scientifico nei sette cantoni della Svizzera romanda.

Parole chiave: Curriculum, PISA-scienze, scuola media, Svizzera romanda 


\section{Science instruction in secondary compulsory education of French-speaking Switzerland with respect to international inquiries on youth scientific culture}

\section{Abstract}

PISA 2006 evaluated the scientific literacy of adolescents from 57 countries. In a period of decreasing interest in science in the OECD countries and because of students' limited scientific skills, it is crucial to involve the school and particularly those of the compulsory years, in developing this literacy. In this article, we survey the science curricula of the of secondary schools in reference to the PISA questions to compare the prescribed scientific education with the demands of the experts about how to reinforce adolescents' interest in science. In this context, we provide an overview of science education in the seven cantons of French speaking Switzerland.

Key words: Curriculum, PISA scientific literacy, compulsory secondary school, French speaking Switzerland 


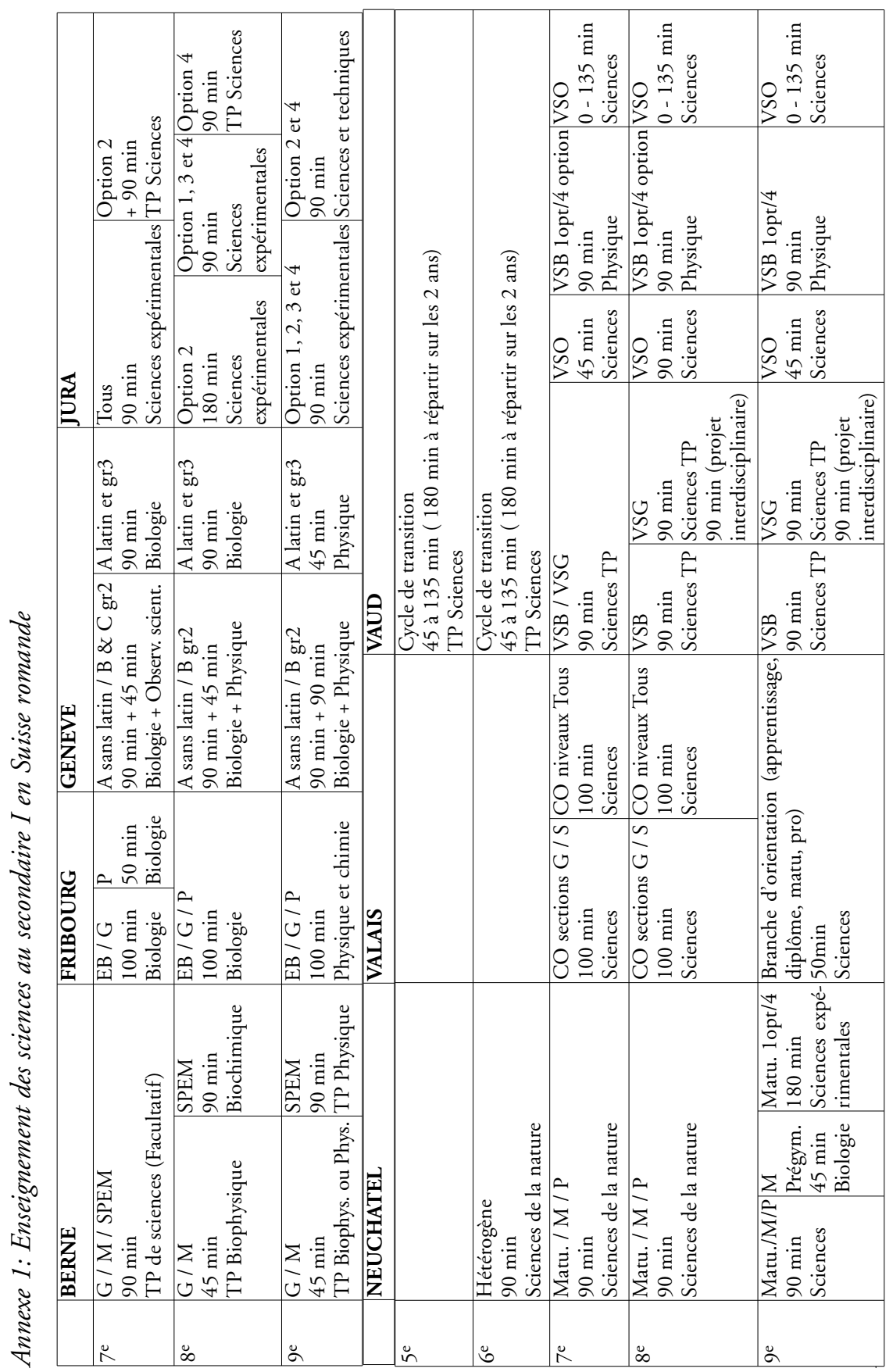


Th e m a

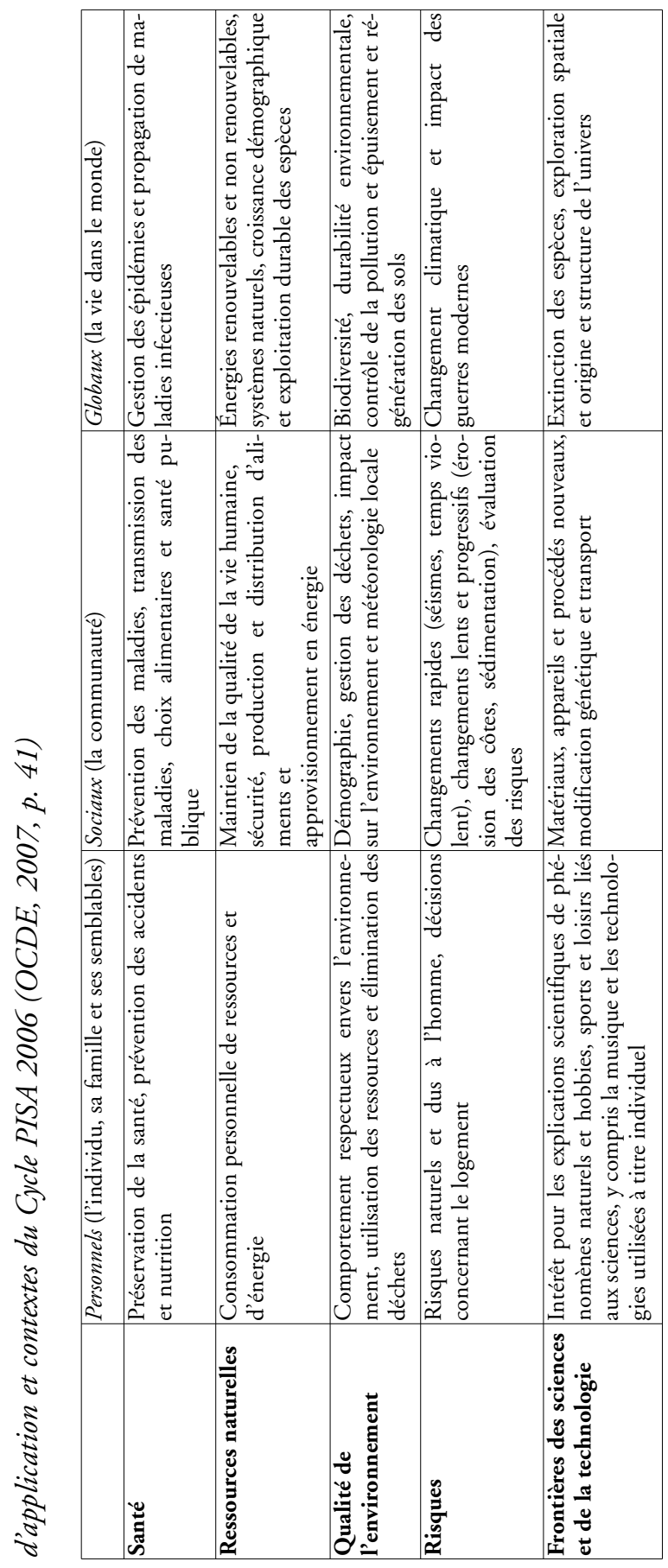

